\title{
Targeted synchronization in an externally driven population of mechanical oscillators
}

Cite as: Chaos 28, 111102 (2018); https://doi.org/10.1063/1.5052652

Submitted: 20 August 2018 . Accepted: 02 November 2018. Published Online: 30 November 2018

Sumit Chhabria, Karen A. Blaha, Fabio Della Rossa, and Francesco Sorrentino
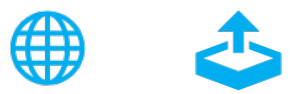

\section{ARTICLES YOU MAY BE INTERESTED IN}

New topological tool for multistable dynamical systems

Chaos: An Interdisciplinary Journal of Nonlinear Science 28, 111101 (2018); https://

doi.org/10.1063/1.5062598

Networks of coupled oscillators: From phase to amplitude chimeras

Chaos: An Interdisciplinary Journal of Nonlinear Science 28, 113124 (2018); https://

doi.org/10.1063/1.5054181

Hierarchical transitions in multiplex adaptive networks of oscillatory units

Chaos: An Interdisciplinary Journal of Nonlinear Science 28, 121101 (2018); https://

doi.org/10.1063/1.5077075

\section{Don't let your writing keep you from getting published!}

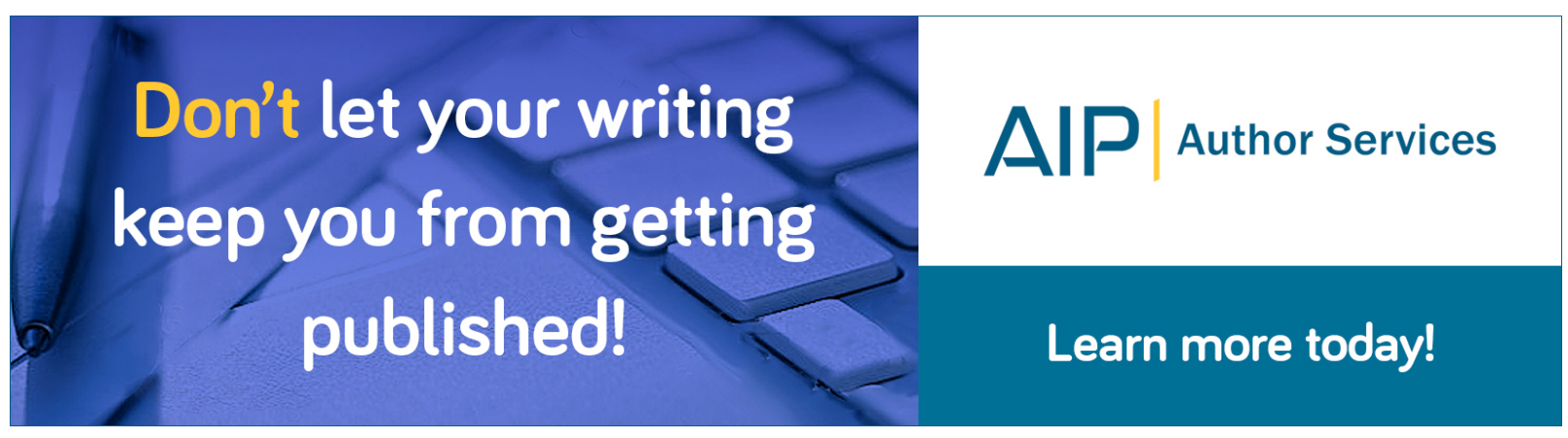




\title{
Targeted synchronization in an externally driven population of mechanical oscillators
}

\author{
Sumit Chhabria, ${ }^{1}$ Karen A. Blaha, ${ }^{1}$ Fabio Della Rossa,,${ }^{1,2}$ and Francesco Sorrentino ${ }^{1,3}$ \\ ${ }^{1}$ Department of Mechanical Engineering, University of New Mexico, Albuquerque, New Mexico 87131, USA \\ ${ }^{2}$ Politecnico di Milano, Piazza Leonardo da Vinci 32, Milano, Italy \\ ${ }^{3}$ Department of Electrical and Computer Engineering, University of New Mexico, Albuquerque, New Mexico \\ 87131, USA
}

(Received 20 August 2018; accepted 2 November 2018; published online 30 November 2018)

\begin{abstract}
We experimentally investigate the synchronization of driven metronomes using a servo motor to impose external control. We show that a driven metronome will only synchronize in a narrow range near its own frequency; when we introduce coupling between metronomes, we can widen the range of frequencies over which a metronome will synchronize to the external input. Using these features, we design a signal to synchronize a population of dissimilar metronomes; separately we design a signal to selectively synchronize a subpopulation of metronomes within a heterogeneous population. Published by AIP Publishing. https://doi.org/10.1063/1.5052652
\end{abstract}

In this work, we experimentally investigate the synchronization of a population of metronomes (interacting or not), when they are driven by an external control signal. We introduce the concept of targeted synchronization, i.e., the possibility of designing a signal that selectively synchronizes only a subpopulation of oscillators within a heterogeneous population. We explore the properties of the driving signal which are needed to observe targeted synchronization in our experimental setup. Applications of this study range from mechanics, where a population of metronomes can be used as an analog frequency identifier for an unknown driving signal, to medical therapies, where external driving signals are often introduced to either synchronize or desynchronize the activity of specific cells in the body.

Oscillator populations driven by external signals arise in numerous biological systems and medical therapies. Pacemaker cells regulate synchrony in the beating of the heart, ${ }^{1,2}$ as do artificial pacemakers. In the case of deep brain stimulation, the signal delivered into the brain with a surgicallyimplanted electrode is thought to disrupt undesired synchrony caused by disease. ${ }^{3}$ The day-night light cycle synchronizes the cells of the suprachiasmatic nucleus. ${ }^{4,5}$ This entrainment of the suprachiasmatic nucleus in turn regulates numerous metabolic processes in both plants and animals. ${ }^{4,6}$ Numerous studies demonstrate the role of the Circadian clock in memory and learning. ${ }^{7}$ Shift workers have long been known to be at increased risk of coronary heart disease. ${ }^{8}$

Many studies explore strategies for the control of populations of coupled oscillators. References 9-11 showed how a complex network can synchronize on a desired time evolution when an external control signal is added to a certain subset of the nodes. Antonsen et al. ${ }^{12}$ and Childs and Strogatz ${ }^{13}$ investigated driving of populations of coupled phase oscillators. The emergence of certain synchronization cluster patterns in a network with symmetries depending upon an external control action was recently studied by Gambuzza et al. ${ }^{14}$
The recent literature has also explored how a uniformlyexperienced coupling can cause multiple qualitatively different behaviors within a population. In the chimera state, parts of a population synchronize while other parts do not: ${ }^{15}$ various work studies chimeras with delayed coupling, ${ }^{16,17}$ chimeras in heterogeneous networks, ${ }^{18}$ and chimeras in nonlocally coupled oscillators. ${ }^{19}$ Certain neurological disorders are associated with specific frequencies within the brain behaving differently; up to $85 \%$ of patients with traumatic brain injury exhibit abnormal delta waves, ${ }^{20,21}$ patients with alcoholism exhibit abnormal delta and theta waves. ${ }^{22}$ Electroencephalogram (EEG) neurofeedback therapy, in which a specific frequency from an EEG is isolated and fed back into the brain, has been shown to be an effective treatment for attention deficit hyperactivity disorder (ADHD); ${ }^{23}$ in mice with Alzheimer's disease, optogenetic driving with gamma frequency light (and only gamma frequency) was shown to reverse harmful amyloid accumulation. ${ }^{24}$

We introduce the concept of targeted synchronization, in which the appropriate choice of driving causes some parts of a population to synchronize while not synchronizing other parts of the population that receive the same driving. The targeted control of elements within a population appears to be of broad interest in a variety of disciplines; however, little experimental work has been performed. Here, we explore this problem experimentally and develop strategies to enforce targeted synchronization, where certain oscillators synchronize and others do not. In particular, we design an external driving signal that causes selective patterns of synchronization.

Since Huygens' observation of the synchronization of two clocks on a beam, ${ }^{25}$ synchronization of mechanical oscillators has been an area of theoretical ${ }^{26}$ and experimental ${ }^{27,28}$ study. Although several studies experimentally investigate coupled mechanical oscillators ${ }^{27-29}$ or experimental driving in other systems, ${ }^{30-32}$ there are few that study driven mechanical oscillators.

We explore the synchronization of metronomes with two kinds of externally provided forcing: direct and indirect. With direct driving, a servo motor is rigidly attached to the 
platform on which the metronomes rest; the platform is thus unaffected by the metronome motion. The metronomes on the directly driven platform do not communicate (see supplementary material, Sec. 1). With indirect driving, the platform receives the input through a spring; the platform moves in response to both the external signal and the motion of the metronomes on the platform. We explore how the driving frequency influences synchronization. We show that metronomes will only synchronize (or in the case of driving, mutually entrain) if driven near their natural frequency; we show that the indirectly driven metronomes synchronize with less external driving because their mutual coupling aids synchrony. Utilizing this observation, we demonstrate that metronomes of different natural frequencies can be selectively synchronized with a properly chosen input signal.

Figure 1 shows our experimental setup. The system consists of 2 platforms; The Directly Driven Platform (DDP) is connected to the servo through rigid joints and the Indirectly Driven Platform (IDP) is connected to the DDP through a spring. Each platform supports up to 4 Wittner Taktell SuperMini metronomes in a line. For further details about the experimental setup, see Refs. 33 and 29. We place UV sensitive dots on both the metronomes and platforms; these dots glow when illuminated by a blacklight in a dark room. We take videos with a typical DSLR camera at 60 frames per second; we then analyze these videos with the Matlab video analysis toolbox to measure the horizontal positions, $X_{i}(t)$, and vertical positions $Y_{i}(t)$, of the metronomes and platform dots.

We drive the DDP with a Quanser SRV02 Rotary Servo Base Unit which we control with Matlab's Simulink package. We can input any driving signal $d(t)$ (i.e., sinusoidal, triangular, arbitrary, etc.) to the motor. We have manually tuned a PID controller up to a cutoff frequency of $2 \mathrm{~Hz}$ (240 BPM) with no steady state error in the ramp response; as a result, the motor arm angle strictly follows the driving signal in the frequency range of interest. The maximum angular displacement of the driven swing arm is $\Theta^{\max }=\arcsin \left[\left(L_{a} / L_{m} \sin (\pi / 8)\right] \sim\right.$ $0.24 \mathrm{rad}$; under these conditions, we use the small angle approximation, giving $\Theta_{1}(t)=0.6 d(t)$.

From the horizontal displacement, $X_{i}(t)-\left\langle X_{i}(t)\right\rangle_{t}$, of each platform dot, we identify the peaks of the oscillatory motion. We compute the platform phase using peak-topeak linear interpolation, $\Phi(t)=2 \pi\left[\left(t-F_{n}\right) /\left(F_{n+1}-F_{n}\right)\right]$, where $F_{n}$ represents the time of peak $n$. To compute the displacement of the metronome arm, we subtract the platform position. We then obtain the phase of each metronome, $\phi_{i}$, with peak identification and linear interpolation.

In a population of metronomes, we define the second Kuramoto order parameter $0 \leq r \leq 1$ of a population of $N$ metronomes as the modulus of the vector that averages twice the phasors of the population, ${ }^{34}$

$$
r(t)=\frac{1}{N}\left|\sum_{i=1}^{N} \exp \left[j 2 \phi_{i}(t)\right]\right|,
$$

where $j=\sqrt{-1}$. Recall that a metronome ticks each time it reaches its maximum horizontal displacement, or twice per oscillation. This symmetry in metronome motion allows both in-phase and anti-phase synchronizations (depending only on

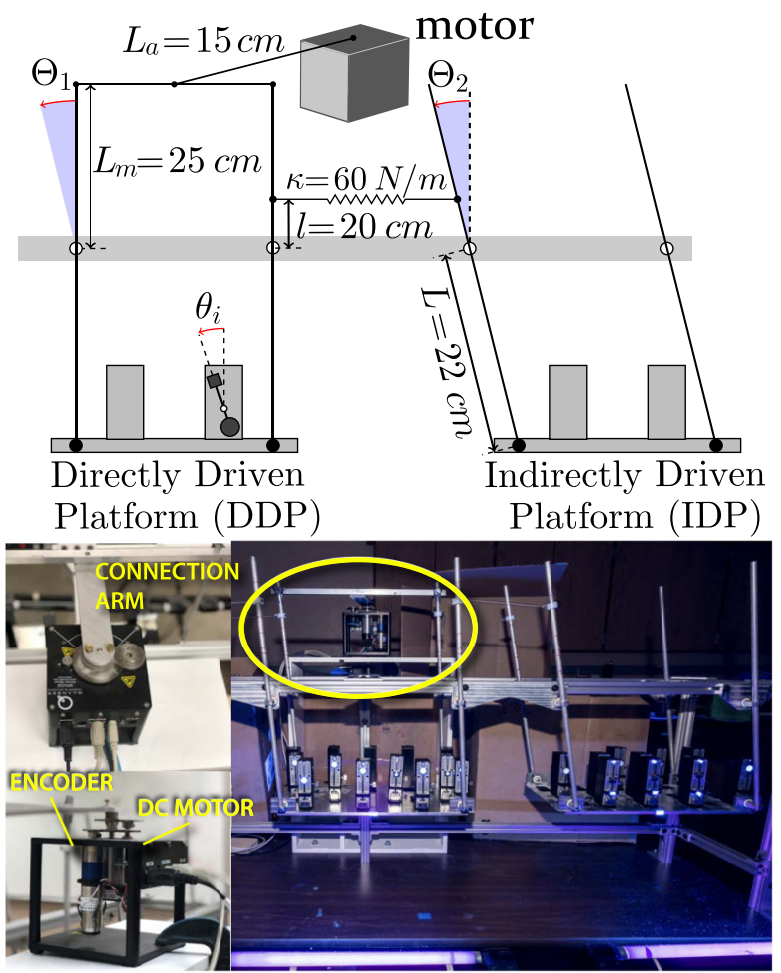

FIG. 1. Experimental Setup. (Top) Schematic of the experimental setup. The total mass of each platform with metronomes is $M=1.6 \mathrm{~kg}$. The left platform is the Directly Driven Platform (DDP), on which the platform exactly follows the input of the servo motor; there is thus no coupling within the population of metronomes. The right platform is the Indirectly Driven Platform (IDP), where servo input enters through a spring; the platform moves in response to both servo input and metronome motion so there is coupling amongst the metronomes. (Bottom) Picture of the experimental setup. The component in the oval is the Quanser SRVO2 Rotary Servo Base Unit.

initial condition); the second Kuramoto order parameter is unity for both in-phase and antiphase synchronizations and thus captures either kind of synchronization. A low value of $r$, on the order of $1 / N$, is consistent with uniformly randomly distributed phases in the range $[0,2 \pi]$. Note that we compute order within the metronomes on each swing, not between swings. The metronomes on the DDP are not coupled; the order reflects the number of single metronomes entraining to the driving. With the order, we can directly compare behavior on the DDP and the IDP. Many studies of driving examine whether the driven oscillator frequency locks with the driving; the order parameter measures if the driven oscillator frequency and phase lock with one another.

We can set the driver to different frequencies and amplitudes. The general equation for the angle $\theta_{i}(t)$ of a metronome on a platform with a time-varying swing angle $\Theta_{j}(t)$ is ${ }^{29}$

$$
\ddot{\theta}_{i}+f\left(\theta_{i}, \dot{\theta}_{i}\right)+\frac{\omega^{2} L}{g} \cos \left(\theta_{i}\right) \ddot{\Theta}_{j}=0,
$$

where $\omega$ is the metronome nominal frequency ( $\omega=$ BPM . $\pi / 60$, since two beats correspond to one oscillation period) and $f$ is the non-linear function describing the metronome motion. To compare experiments with different metronome frequencies and different driving signal frequencies, we tune the amplitude of the driving signal so that the maximum effect of the driver on the metronomes is equal in each swing. 
Experiments on the DDP and the IDP are performed separately so that the driving is tuned for the platform of interest. We choose driving amplitudes for each swing so that the maximum acceleration moduli are equal, $\ddot{\Theta}_{1}^{\max }=\ddot{\Theta}_{2}^{\max }$. For the DDP, a driving signal of $d(t)=A \sin (\Omega t)$ gives

$$
\ddot{\Theta}_{1}^{\max }=\max _{t}\left|0.6 A \Omega^{2} \sin (\Omega t)\right|=0.6 A \Omega^{2} .
$$

For the IDP, neglecting the effect of the metronomes motion on the IDP, the swing angle $\Theta_{2}(t)$ follows

$$
\ddot{\Theta}_{2}+\frac{v_{s}}{M} \dot{\Theta}_{2}+\frac{g}{L} \Theta_{2}+\frac{\kappa}{M} \frac{l}{L^{2}}\left(\Theta_{2}-\Theta_{1}\right)=0,
$$

where $v_{s}=0.27 \mathrm{~kg} \mathrm{~s}^{-1}$ is a damping parameter, obtained by the time needed for the free-swinging platform to stop its motion after an initial perturbation $(\sim 30 \mathrm{~s})$. Since $\Theta_{1}(t)$ is proportional to the servo input $d(t)$, we can compute the transfer function of the indirectly driven swing angle as

$$
G_{\Theta_{1} \rightarrow \Theta_{2}}(\Omega)=\frac{\kappa l}{M L^{2}(i \Omega)^{2}+L^{2} v_{s}(i \Omega)+M L g+\kappa l} .
$$

$\left|G_{\Theta_{1} \rightarrow \Theta_{2}}(\Omega)\right|$ is the ratio between the energies that the driver transmits to the indirectly driven platform and the directly driven platform. In the top panel of Fig. 2, we show the variation of $\left|G_{\Theta_{1} \rightarrow \Theta_{2}}(\Omega)\right|$ as with variation in frequency; note the resonance peak at $\omega=160 \mathrm{BPM}$. To limit the effect of this peak, in what follows we use driver frequencies smaller than $120 \mathrm{BPM}$. We choose $72 \mathrm{BPM}$ as the minimum frequency, since the metronomes' frequencies in this range can be uniformly tuned with a step of 4 BPM. In the bottom panel of Fig. 2, we report the IDP frequency response in the range of analysis; experimental data acquired to validate the theoretical results is shown by red circles. Note that another small resonance peak (not predicted by the linearized model) is present at $80 \mathrm{BPM}$; we use these experimental values of $\left|G_{\Theta_{1} \rightarrow \Theta_{2}}(\Omega)\right|$ to calculate the driving amplitude for later experiments. The peak is at $80 \mathrm{BPM}$, exactly half the frequency of the major resonance peak at $160 \mathrm{BPM}$, and can be explained looking at the nonlinear driven pendulum model. ${ }^{35}$

We explore the emergence of synchrony in response to a driving force on the DDP and the IDP. We manually start 96 BPM metronomes in a roughly synchronous condition. Different initial conditions do not affect the results; when synchrony occurs, this state includes some metronomes inphase and some anti-phase with respect to the platform. We apply sinusoidal driving, scanning over a range of frequencies $(\Omega=88-104$ BPM in increments of 4 BPM) and maximum angular accelerations $\left(\ddot{\Theta}_{i}^{\max }\right.$ from $2 \mathrm{rad} \mathrm{s}^{-2}$ to $10 \mathrm{rad} \mathrm{s}^{-2}$ ). We report results for a larger range of driving frequencies in Sec. 3 of the supplementary material.

Figure 3 shows the Kuramoto order of the metronome population time-averaged over the last $30 \mathrm{~s}$ of the experiment (each experiment is $2 \mathrm{~min}$ long). For each experiment, we also report either the time needed to achieve a constant Kuramoto order or the minimum and maximum order parameter value when it fails to reach a steady order value. The response of the DDP, shown in the left of Fig. 3, demonstrates that the population of metronomes acts as a frequency selector, i.e., we only observe frequency and phase synchronization when the driver and metronome frequencies are the same (96 BPM).

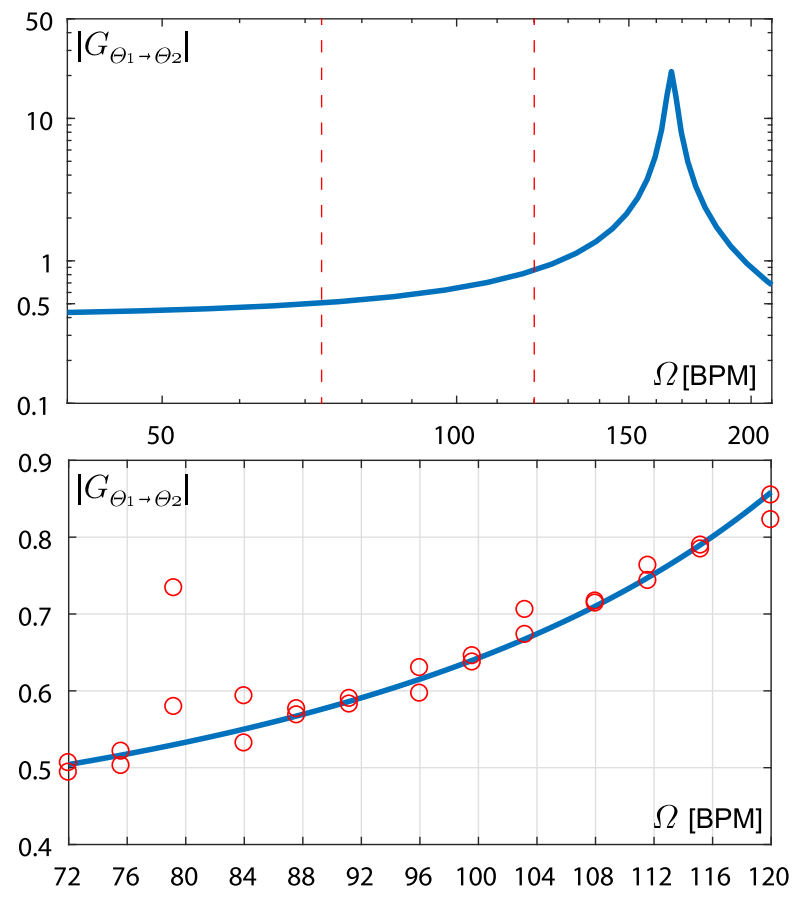

FIG. 2. Frequency response of the indirectly driven platform. (Top) Calculated transmitted energy, $G_{\Theta_{1} \rightarrow \Theta_{2}}(\Omega)$, for full range of possible metronome frequencies. The dashed red lines indicate the range of frequencies plotted in the bottom panel. (Bottom) Diagram of frequencies between 72 BPM and $120 \mathrm{BPM}$. The red circles are experimental data.

When synchronization is achieved, the Kuramoto order value is around 0.98 (white in the figure); the metronome phases synchronize in less than $20 \mathrm{~s}$. Note that there is a lower threshold (black dotted line in the figure) in the acceleration, $\ddot{\Theta}_{i}^{\max }$, needed to achieve global synchronization. Below this threshold, the Kuramoto order of the metronome population varies over time; the amplitude of this variation grows with decreasing forcing.

When we drive the DDP with a different frequency $(\Omega \neq$ 96 BPM), we never obtain a constant Kuramoto order greater than 0.8 for the range shown in the figure. The constant subunity values arise from small phase offsets; the metronomes oscillate at roughly the driving frequency but maintain phase offsets not equal to zero or $\pi$. For example, at $\Omega=100$ BPM, all the metronomes tick within $0.15 \mathrm{~s}$ (for $\ddot{\Theta}_{1}^{\max }=8 \mathrm{rad} \mathrm{s}^{-2}$ ) and $0.08 \mathrm{~s}$ (for $\ddot{\Theta}_{1}^{\max }=10 \mathrm{rad} \mathrm{s}^{-2}$ ) of one another, while $0.6 \mathrm{~s}$ elapses between two ticks for a 100 BPM metronome. Further increasing $\ddot{\Theta}_{1}^{\max }$ to $12 \mathrm{rad} \mathrm{s}^{-2}$ (not in figure) at this frequency produces synchronization of the population at $100 \mathrm{BPM}$; note that to achieve synchronization at this frequency, we need twice the angular acceleration required for $\Omega=96 \mathrm{BPM}$. We observe periodic oscillations of the order when the acceleration given by the driver is less than the threshold. In this case, the metronome amplitudes oscillate quasi-periodically while the frequency stays roughly at the natural value (96 BPM), see supplementary material, Sec. 4. For $\Omega=88 \mathrm{BPM}$ and $\ddot{\Theta}_{1}^{\max }=10 \mathrm{rad} \mathrm{s}^{-2}$, we observed amplitude death ${ }^{36-38}$ in one to two metronomes over several repetitions, see Sec. 4 of the supplementary material.

When we compare the DDP (left panel) and the IDP (right panel), we see that the exchange of energy between the 

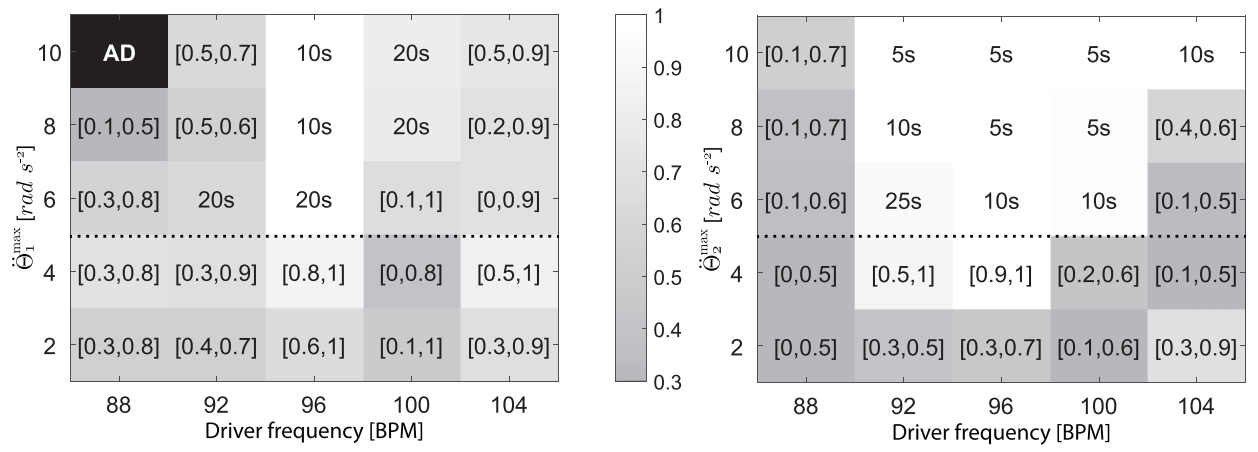

FIG. 3. Average Kuramoto order for four $96 \mathrm{BPM}$ metronomes with different driving frequency, $\Omega$, and maximum driven acceleration, $\ddot{\Theta}_{i}^{\max }$. (Left) metronomes on the DDP, (Right) metronomes on the IDP. For each experiment we report either the time for the Kuramoto order parameter to reach a constant value or the maximum and minimum values of the Kuramoto order as it varies with time (reported in brackets). AD stands for amplitude death ${ }^{36}$ and means that the metronome amplitude goes to zero for the experiment. The black dotted lines indicate the angular acceleration threshold required to synchronize at $\Omega=\omega$.

metronomes and the platform which is possible on the IDP facilitates the mutual entrainment of the population to driving. Although the metronomes exchange so little energy in the absence of driving that they cannot synchronize, this small difference between the IDP and the DDP produces a large qualitative difference in synchronization between the two.

When synchronization is not achieved, the average order is lower and the oscillations of the metronomes are always quasi-periodic.

The IDP exhibits synchrony even with a driving frequency different than but close to that of the metronomes. The synchronization tongue ${ }^{39}$ of the IDP is asymmetrical; it is easier to synchronize the metronomes when the driving is faster (i.e., when $\Omega>\omega$ ). This asymmetry was also present on the DDP, although less pronounced.

The results we present are not specific to the metronome frequencies we have selected. Figure 4 reports the order observed in a population of 4 metronomes with 13 different natural frequencies (the available metronomes settings between 72 BPM and 120 BPM) driven with a signal just above the synchronization threshold for frequencies that differ within $\pm 8 \mathrm{BPM}$ of the metronomes frequency. From Fig. 3, we see that synchronization begins at $\ddot{\Theta}_{1}^{\max }=\ddot{\Theta}_{2}^{\max }=$ $6 \mathrm{rad} / \mathrm{s}^{2}$ (see Table 2 in the supplementary material for the values of $A$ used); we use this driving to drive metronomes of different frequencies. For the DDP, we omit $\omega=72$ BPM and for the IDP, we omit $\omega=72-84$ BPM because the amplitude of the computed driving signal exceeded the maximum amplitude the servo-motor can generate. Again, for all the analyzed frequencies, we only observe total frequency and phase entrainment (average Kuramoto order $>0.96$ ) in the DDP when the driver and the metronome frequencies are the same, thus confirming the capability of the metronomes' population to act as a frequency selector. Note that metronome selectivity works better at higher frequencies, since the higher frequency curves exhibit sharper peaks (see Fig. 4). On the IDP, we again see that metronomes synchronize across a wider range of driving frequencies.

To better underline this property, we place 4 metronomes with 4 different natural frequencies (104 BPM, 108 BPM, 112 BPM, and 116 BPM) and impose a driving signal with frequency $110 \mathrm{BPM}$ and an amplitude which slowly increases with time for a total of $300 \mathrm{~s}$. Figure 5 gives the $20 \mathrm{~s}$ moving average of the order of this population of metronomes both on the DDP (thick cyan curve) and on the IDP (thin red curve), as a function of the maximum acceleration of each platform $\ddot{\Theta}_{i}^{\max }$. The acceleration needed to synchronize the population on the IDP is less than for the DDP. In the IDP, we see that the order jumps twice, first when the three fastest metronomes synchronize (around $\ddot{\Theta}_{2}^{\max }=5 \mathrm{rad} \mathrm{s}^{-2}$ ), and second when the slowest metronome joins the other three (around $\ddot{\Theta}_{2}^{\max }=$ $11 \mathrm{rad} \mathrm{s}^{-2}$ ). We expect the slowest metronome to synchronize with the most effort; slower metronomes require more acceleration to experience the same magnitude of forcing in Eq. (2). We also saw in Fig. 3 that the synchronization region is asymmetric toward faster frequencies. On the other hand, the DDP remains disordered until around $\ddot{\Theta}_{1}^{\max }=12 \mathrm{rad} \mathrm{s}^{-2}$.

Conversely, one might prefer to exploit the heterogeneity of a system. Consider a population composed of two subpopulations with different frequencies; can we drive the

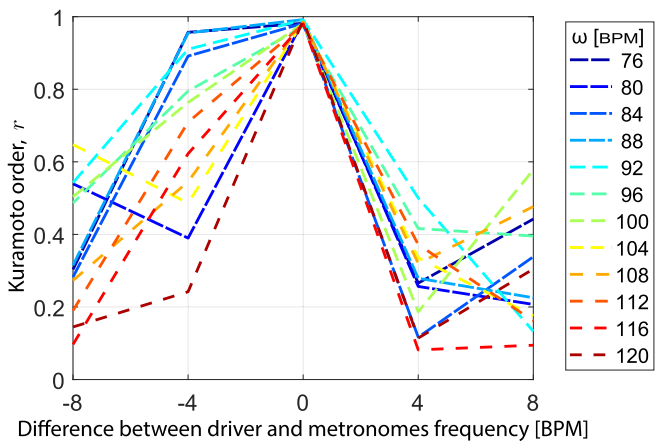

Difference between driver and metronomes frequency [BPM]

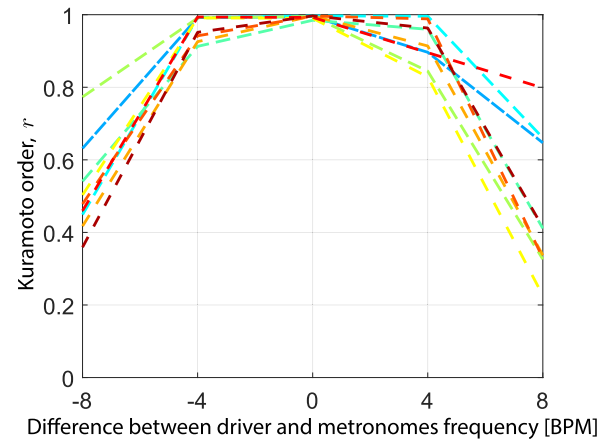

FIG. 4. Average Kuramoto order of a population of four metronomes. On each line, the population is tuned at a different natural frequency (see legend). The driving signal frequency is in the neighborhood of the metronomes natural frequency, with amplitude selected in order to be just above the same-frequency synchronization threshold. DDP and IDP are in the left and right panel, respectively. 


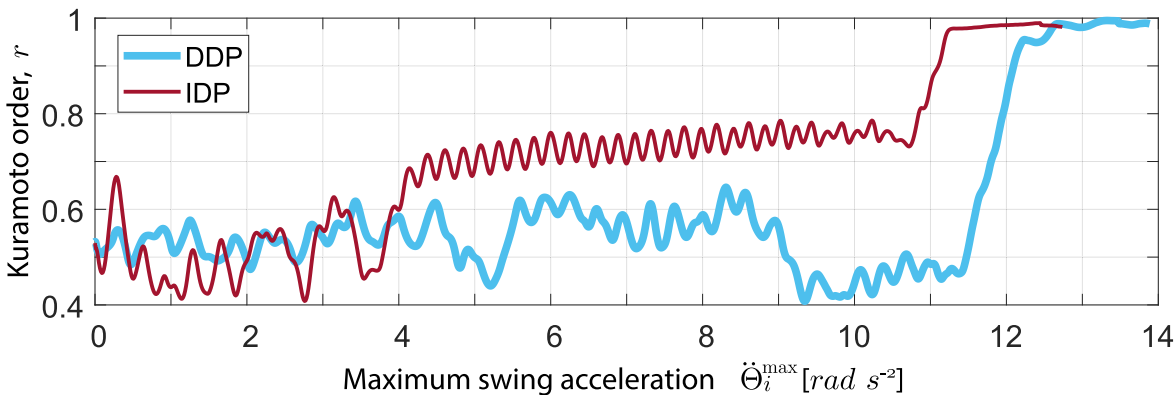

FIG. 5. Average Kuramoto order of a population of 4 different metronomes (natural frequencies ranging from $104 \mathrm{BPM}$ to $116 \mathrm{BPM}$ ) driven with a signal with amplitude that slowly increases in time. system in order to selectively synchronize each or both the subpopulations? Since the DDP behaves more strongly as a frequency selector, we place on it 4 metronomes with 2 different natural frequencies (two of them at $100 \mathrm{BPM}$ and the other two at $116 \mathrm{BPM})$. We then drive the system with the following signal:

$$
d(t)= \begin{cases}0, & \text { if } t \in[30,60], \\ 0.3 \sin (116 / 1202 \pi t), & \text { if } t \in[30,90], \\ \sin (100 / 1202 \pi t), & \text { if } t \in[90,150], \\ 0.3 \sin (116 / 1202 \pi t) & \\ +\sin (100 / 1202 \pi t), & t>150 .\end{cases}
$$

The result of this experiment is reported in the upper panel of Fig. 6.

When only one driving signal is present, the corresponding population of metronomes synchronizes. With both harmonics, both subpopulations synchronize, each at its own natural frequency (i.e., the metronomes inside each subpopulation are synchronized, but those from two different subpopulations are not). We chose the amplitude of the driving signals so that the acceleration from driving in Eq. (2), $\ddot{\Theta}_{i}^{\max }$, is constant. We observe that, in order for the two populations to synchronize separately, we need the two natural frequencies of the metronomes to be at least 12 BPMs apart (roughly $10 \%$ of the natural frequency). We perform the same experiment on the IDP; see the lower panel of Fig. 6. We change the amplitudes of the driving signal according to the experimental values from the right panel of Fig. 2 in order to keep the same maximum acceleration of the two platforms. Although we observe targeted synchronization on the IDP too, the population takes longer to synchronize and is less synchronized after this longer transient.

We show that the entrainment of driven metronomes varies depending upon the presence of coupling within the population. With no population coupling (on the DDP), entrainment occurs only when the natural metronome frequency is close to the driving frequency; when we allow population coupling (on the IDP), entrainment occurs when the driving frequency differs by a certain amount from the natural frequency. We have experimentally verified that these results are robust even to changes in the waveforms of the driving signal (i.e., triangular, square, etc.). The entrainment to driving (as on the DDP) is typical for forced nonlinear oscillators; similar dynamics occurs in predator-prey models, ${ }^{40}$ love dynamics, ${ }^{41}$ and experimental optics, ${ }^{42}$ among others. However, the synchronization range of the metronomes is unusually narrow (see supplementary material, Sec. 3) and shrinks if we add more metronomes. This observation suggests the possibility of using a metronomes population as an analog frequency identifier for an unknown driving signal.

We chose to keep the model for metronome nonlinearity in Eq. (2) generic, $f\left(\theta_{i}, \dot{\theta}_{i}\right)$, as we found that the Van der Pol-based model, which is generally used when studying metronome synchronization, ${ }^{28,29,33}$ was not suitable for
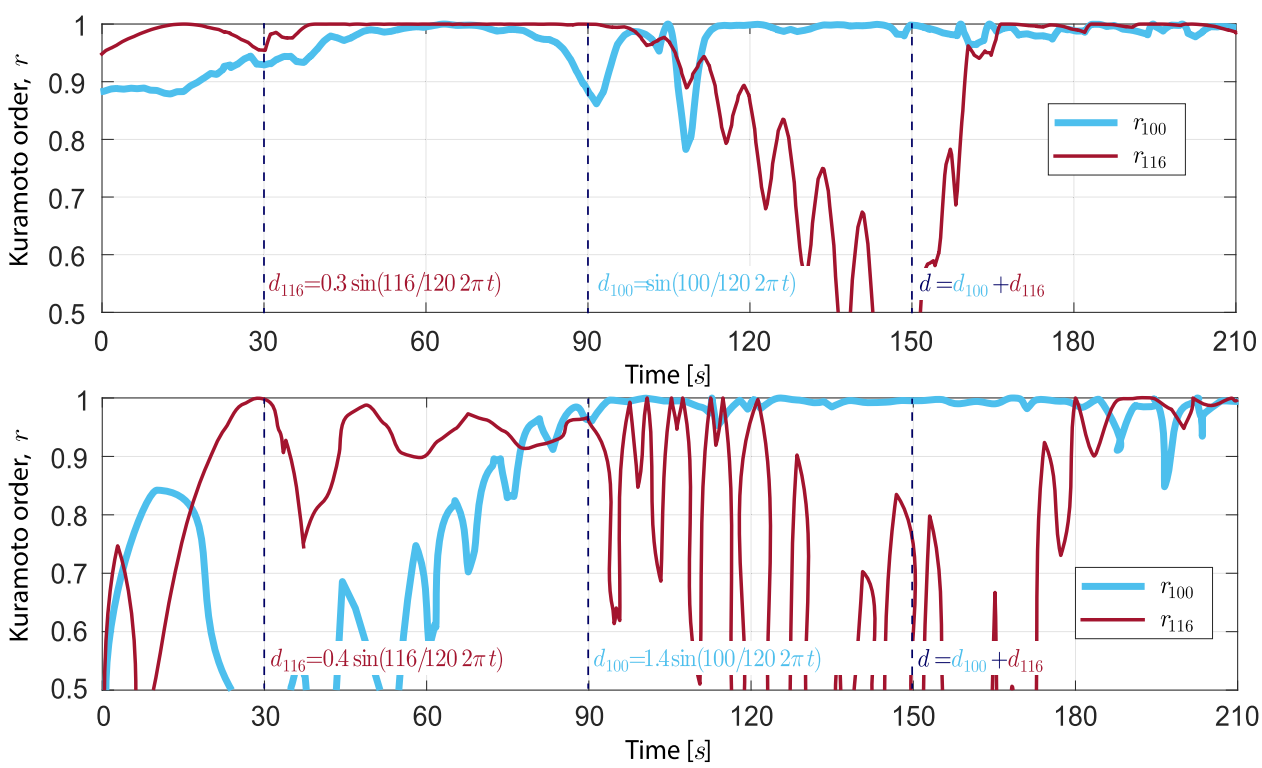

FIG. 6. Kuramoto order of two populations composed by two 100 BPM (green) and two 116BPM (red) metronomes on the DDP (upper panel) and on the IDP (lower panel). The driving signal changes at $t=30,90,150$ as written in the figure. 
several reasons: (1) The model frequently predicts driven solutions with amplitudes of $\pi / 2$, larger than physically possible in the metronome. (2) The model cannot predict amplitude death, which we observed experimentally (see supplementary material, Sec. 4.2). (3) The model does not predict the amplitude and frequency variation we observe in the uncoupled metronome (see supplementary material, Sec. 1). (4) We did not find a satisfactory fit with the model when the driving frequency equaled the metronome frequency (when $\Omega=\omega$ ). In Sec. 3 of the supplementary material, we also show that the Van der Pol model predicts much broader entrainment to driving than we experimentally observe. Theoretical studies on metronomes sychronization should thus rely on more physically-grounded models, such as the ones proposed in Kapitaniak et al. $^{43}$

In this paper we have characterized how a population of nonlinear oscillators responds to external driving, both with and without internal coupling. This controlled experiment is analogous to a variety of biological examples; for example, the entrainment of the cells of the suprachiasmatic nucleus (SCN) to the external forcing of the sun is responsible for the circadian rhythm. Like the metronomes, the synchronization of the circadian rhythm occurs only near the natural frequency of the SCN cells. ${ }^{5,44}$ We have described how the environment of an experimental nonlinear oscillator can influence its synchronization to an externally-imposed driving signal. We have also explored the idea of targeted synchronization and show how the presence of internal coupling within a heterogeneous population can interfere with the ability of a targeted group to synchronize. Many medical therapies apply an external signal to populations with coupling; that internal coupling is not always fully known. Further characterization of the influence of internal coupling on synchronization may enable the improvement of such therapies.

\section{SUPPLEMENTARY MATERIAL} ing on:

See supplementary material that has four sections focus-

1. Metronome behaviors and a quantification of their interaction through the DDP and the IDP.

2. The computation of the driving amplitude $A$ in order to maintain the maximum platform acceleration $\Theta_{i}^{\max }$ constant.

3. A discussion on the frequency selectivity observed on the DDP.

4. A description of the interesting nonlinear behaviors (quasi-periodicity, amplitude death) described throughout the paper.

\section{ACKNOWLEDGMENTS}

This work is supported by the National Science Foundation through NSF Grant Nos. CMMI- 1400193 and CMMI1727948, ONR Award No. N00014-16-1-2637, as well as HDTRA1-13-1-0020.

${ }^{1}$ L. Glass and M. C. Mackey, From Clocks to Chaos: The Rhythms of Life (Princeton University Press, 1988).

${ }^{2}$ D. C. Michaels, E. P. Matyas, and J. Jalife, Circ. Res. 61, 704 (1987).
${ }^{3}$ A. Eusebio, W. Thevathasan, L. D. Gaynor, A. Pogosyan, E. Bye, T. Foltynie, L. Zrinzo, K. Ashkan, T. Aziz, and P. Brown, J. Neurol. Neurosurg. Psychiatry 82, 569 (2011).

${ }^{4}$ S. Yamaguchi, H. Isejima, T. Matsuo, R. Okura, K. Yagita, M. Kobayashi, and H. Okamura, Science 302, 1408 (2003).

${ }^{5}$ C. A. Czeisler, J. S. Allan, S. H. Strogatz, J. M. Ronda, R. Sanchez, C. D. Rios, W. O. Freitag, G. C. Richardson, and R. E. Kronauer, Science 233, 667 (1986).

${ }^{6}$ H. Fukuda, N. Nakamichi, M. Hisatsune, H. Murase, and T. Mizuno, Phys. Rev. Lett. 99, 098102 (2007).

${ }^{7}$ B. L. Smarr, K. J. Jennings, J. R. Driscoll, and L. J. Kriegsfeld, Behav. Neurosci. 128, 283 (2014).

${ }^{8}$ G. Kuhn, Ann. Emergency Med. 37, 88 (2001).

${ }^{9}$ X. F. Wang and G. Chen, IEEE Trans. Circuits Syst. I Fundam. Theory Appl. 49, 54 (2002).

${ }^{10}$ X. F. Wang and G. Chen, Phys. A Stat. Mech. Appl. 310, 521 (2002).

${ }^{11}$ F. Sorrentino, M. Di Bernardo, F. Garofalo, and G. Chen, Phys. Rev. E 75, 046103 (2007).

${ }^{12}$ T. Antonsen, Jr., R. Faghih, M. Girvan, E. Ott, and J. Platig, Chaos 18, 037112 (2008).

${ }^{13}$ L. M. Childs and S. H. Strogatz, Chaos 18, 043128 (2008).

${ }^{14}$ L. V. Gambuzza, M. Frasca, and V. Latora, "Distributed control of synchronization of a group of network nodes," IEEE Trans. Automat. Cont. (published online).

${ }^{15}$ D. M. Abrams and S. H. Strogatz, Phys. Rev. Lett. 93, 174102 (2004).

${ }^{16}$ O. E. Omelchenko, Y. L. Maistrenko, and P. A. Tass, Phys. Rev. Lett. 100, 044105 (2008).

${ }^{17}$ L. Larger, B. Penkovsky, and Y. L. Maistrenko, Phys. Rev. Lett. 111(5), 054103 (2013).

${ }^{18}$ C. R. Laing, Chaos 19, 013113 (2009).

${ }^{19}$ S. Nkomo, M. R. Tinsley, and K. Showalter, Phys. Rev. Lett. 110, 244102 (2013).

${ }^{20}$ C. Reis, Y. Wang, O. Akyol, W. M. Ho, R. Applegate, G. Stier, R. Martin, and J. H. Zhang, Int. J. Mol. Sci. 16, 11903 (2015).

${ }^{21}$ M.-X. Huang, C. W. Huang, A. Robb, A. Angeles, S. L. Nichols, D. G. Baker, T. Song, D. L. Harrington, R. J. Theilmann, and R. Srinivasan et al., Neuroimage 84, 585 (2014).

${ }^{22}$ I. M. Colrain, S. Turlington, and F. C. Baker, Sleep 32, 1341 (2009).

${ }^{23}$ M. Arns, S. de Ridder, U. Strehl, M. Breteler, and A. Coenen, Clin. EEG Neurosci. 40, 180 (2009).

${ }^{24}$ H. F. Iaccarino, A. C. Singer, A. J. Martorell, A. Rudenko, F. Gao, T. Z. Gillingham, H. Mathys, J. Seo, O. Kritskiy, and F. Abdurrob et al., Nature 540, 230 (2016).

${ }^{25} \mathrm{C}$. Huygens, Oeuvrescomplètes (M. Nijhoff, 1897), Vol. 7.

${ }^{26}$ K. Czoczyński, P. Perlikowski, A. Stefański, and T. Kapitaniak, Chaos 21, 023129 (2011).

${ }^{27}$ M. Bennett, M. F. Schatz, H. Rockwood, and K. Wiesenfeld, Proc. Math. Phys. Eng. Sci. 458 563-579 (2002).

${ }^{28}$ J. Pantaleone, Am. J. Phys. 70, 992 (2002).

${ }^{29}$ E. A. Martens, S. Thutupalli, A. Fourrière, and O. Hallatschek, Proc. Natl. Acad. Sci. 110, 10563 (2013).

${ }^{30}$ G. Heinrich, M. Ludwig, J. Qian, B. Kubala, and F. Marquardt, Phys. Rev. Lett. 107, 043603 (2011).

${ }^{31}$ J. Simonet, M. Warden, and E. Brun, Phys. Rev. E 50, 3383 (1994).

${ }^{32}$ K. A. Blaha, A. S. Pikovsky, M. G. Rosenblum, M. T. Clark, C. G. Rusin, and J. L. Hudson, Phys. Rev. E 84, 046201 (2011).

${ }^{33}$ K. Blaha, R. J. Burrus, J. L. Orozco-Mora, E. Ruiz-Beltrán, A. B. Siddique, V. Hatamipour, and F. Sorrentino, Chaos 26, 116307 (2016).

${ }^{34}$ H. Daido, Prog. Theor. Phys. 88, 1213 (1992).

${ }^{35}$ E. I. Butikov, J. Phys. A Math. Gen. 35, 6209 (2002).

${ }^{36}$ G. B. Ermentrout and N. Kopell, SIAM J. Appl. Math. 50, 125 (1990).

${ }^{37}$ D. V. Ramana Reddy, A. Sen, and G. L. Johnston, Phys. Rev. Lett. 85, 3381 (2000).

${ }^{38}$ Y. Zhai, I. Z. Kiss, and J. L. Hudson, Phys. Rev. E 69, 026208 (2004).

${ }^{39}$ A. Pikovsky, M. Rosenblum, J. Kurths, and J. Kurths, Synchronization: A Universal Concept in Nonlinear Sciences (Cambridge University Press, 2003), Vol. 12.

${ }^{40}$ A. Gragnani and S. Rinaldi, Bull. Math. Biol. 57, 701 (1995).

${ }^{41}$ S. Rinaldi, F. D. Rossa, F. Dercole, A. Gragnani, and P. Landi, Modeling Love Dynamics (World Scientific, 2016).

${ }^{42}$ K. Huang and M. Hossein-Zadeh, Opt. Express 26, 8275 (2018).

${ }^{43}$ M. Kapitaniak, K. Czolczynski, P. Perlikowski, A. Stefański, and T. Kapitaniak, Phys. Rep. 517, 1 (2012).

${ }^{44}$ J. N. Mills, D. S. Minors, and J. M. Waterhouse, J. Physiol. 240, 567 (1974). 